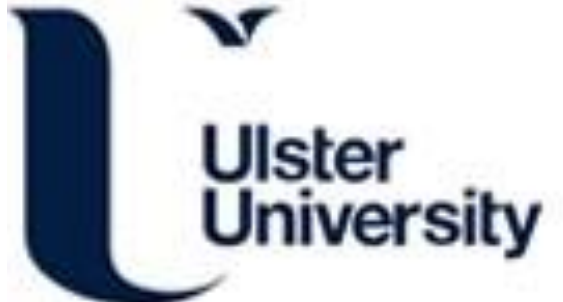

\section{Audio Content Analysis for Unobtrusive Event Detection in Smart Homes}

Vafeiadis, A., Votis, K., Giakoumis, D., Tzovaras, D., Chen, L., \& Hamzaoui, R. (2020). Audio Content Analysis for Unobtrusive Event Detection in Smart Homes. Engineering Applications of Artificial Intelligence, 89, [103226]. https://doi.org/10.1016/j.engappai.2019.08.020

Link to publication record in Ulster University Research Portal

\section{Published in:}

Engineering Applications of Artificial Intelligence

\section{Publication Status:}

Published (in print/issue): 31/03/2020

DOI:

10.1016/j.engappai.2019.08.020

\section{Document Version}

Author Accepted version

\section{General rights}

Copyright for the publications made accessible via Ulster University's Research Portal is retained by the author(s) and / or other copyright owners and it is a condition of accessing these publications that users recognise and abide by the legal requirements associated with these rights.

\section{Take down policy}

The Research Portal is Ulster University's institutional repository that provides access to Ulster's research outputs. Every effort has been made to ensure that content in the Research Portal does not infringe any person's rights, or applicable UK laws. If you discover content in the Research Portal that you believe breaches copyright or violates any law, please contact pure-support@ulster.ac.uk. 


\title{
Audio Content Analysis for Unobtrusive Event Detection in Smart Homes
}

\author{
Anastasios Vafeiadis ${ }^{\mathrm{a}, *}$, Konstantinos Votis $^{\mathrm{a}}$, Dimitrios Giakoumis ${ }^{\mathrm{a}}$, Dimitrios \\ Tzovaras $^{\mathrm{a}}$, Liming Chen ${ }^{\mathrm{b}}$, Raouf Hamzaoui ${ }^{\mathrm{b}}$ \\ ${ }^{a}$ Center for Research and Technology Hellas - Information Technologies Institute, \\ Thessaloniki, Greece \\ ${ }^{b}$ Faculty of Computing, Engineering and Media, De Montfort University, Leicester, UK
}

\begin{abstract}
Environmental sound data are multi-source, heterogeneous, and varying in time. Many systems have been proposed to process such data for event detection in ambient assisted living applications. Typically, these systems use feature extraction, selection, and classification. However, despite major advances, several important questions remain unanswered, especially in real-world settings. This paper contributes to the body of knowledge in the field by addressing the following problems for ambient sounds recorded in various real-world kitchen environments: 1) which features and which classifiers are most suitable in the presence of background noise? 2) what is the effect of signal duration on recognition accuracy? 3) how do the signal-to-noise-ratio and the distance between microphone and audio source affect the recognition accuracy in an environment in which the system was not trained? We show that for systems that use traditional classifiers, it is beneficial to combine gammatone frequency cepstral coefficients and discrete wavelet transform coefficients and to use a gradient boosting classifier. For systems based on deep learning, we consider 1D and 2D Convolutional Neural Networks (CNN) using mel-spectrogram energies and mel-spectrograms images, as inputs, respectively and show that the 2D CNN outperforms the 1D CNN. We obtained competitive classification results for two
\end{abstract}

\footnotetext{
* Corresponding author

Email address: anasvaf@iti.gr (Anastasios Vafeiadis)
} 
such systems. The first one, which uses a gradient boosting classifier, achieved an F1-Score of $90.2 \%$ and a recognition accuracy of $91.7 \%$. The second one, which uses a 2D CNN with mel-spectrogram images, achieved an F1-Score of $92.7 \%$ and a recognition accuracy of $96 \%$.

Keywords: Smart homes; ambient assisted living; audio signal processing; feature extraction; feature selection; deep learning

\section{Introduction}

Smart home-based ambient assisted living Information and Communications Technology (ICT) solutions can allow the elderly to remain in their own homes for longer and live independently [1. Research on ICT solutions for ambient 5 assisted living has intensified over the last decades considerably, due to the emergence of affordable powerful sensors and progress in artificial intelligence [2, 3, 4.

Diverse human activity recognition (HAR) systems that monitor daily activities to identify abnormal behavior have been proposed for ambient assisted living applications [5, 6].

One common approach to automated HAR uses portable sensors such as accelerometers and gyroscopes [7, 8. However, these sensors require cooperation of the subject, may restrict body movement, and are energy constrained [9, 10]. Another approach relies on computer vision [11, 12. However, privacy concerns 15 are hindering its adoption. A further approach is based on audio processing. Features are extracted from the environmental sounds and classifiers are used to recognize the corresponding human activity [13, 14, 15].

While several audio-based HAR systems have been proposed, a number of important questions remain unanswered:

- which features and which classifiers are most suitable in the presence of background noise?

- what is the effect of the duration of the signal segment used for classification on recognition accuracy? Decreasing the segment duration decreases 
the response time of the system but may harm its recognition accuracy. of multiple events within the same sound segment;

- how do the signal-to-noise-ratio (SNR) and the distance between the microphone and audio source affect the recognition accuracy in a new environment (i.e., one which was not used to train the classifier)?

Our paper answers these questions for a real-world indoor kitchen environment where large audio datasets are captured and processed to train classifiers. Two representative acoustic event detection (AED) approaches are studied. The first one extracts time and frequency features and uses a traditional classifier. We compared various features and classifiers and showed that the best results were obtained with hybrid time-frequency features, together with a gradient boosting classifier. Our best system achieved an F1-score of $90.2 \%$ and a recognition accuracy of $91.7 \%$. The second system uses mel-spectrogram images of the audio signals as input to a $2 \mathrm{D} \mathrm{CNN}$. We showed that compared to a $1 \mathrm{D} \mathrm{CNN}$ that applies max-pooling to only one dimension, applying max pooling to both 40 dimensions of the input (time and frequency) reduces the dimensionality in a more uniform manner, yielding more salient features with each consecutive convolutional-max pooling operation. This approach achieved a recognition accuracy of $96 \%$ and an F1-Score of $92.7 \%$. Additionally, we observed that in a real-world environment the recognition accuracy for some of the selected classes decreased with increasing duration of the audio segment and did not significantly improve when the duration was greater than $3 \mathrm{~s}$. This was due to overlapping sounds that occurred in the kitchen environment (e.g., kitchen faucet running, while the user picks a plate to wash). In a scenario where the data needs to be captured and processed on a system on chip device (e.g., Raspberry Pi), the small trade off between the memory size of the data and the recognition accuracy, is crucial. Finally, since real-world environments typically include noise, we studied the effect of the SNR and distance between the microphone and the target audio event on the recognition accuracy. For events such as using 
the mixer and the utensils (forks, spoons, knives), the recognition accuracy was high despite the background noise of a kitchen fan and a refrigerator. The high frequencies of these events could mask the low frequencies of the background noise. On the other hand, we noticed a drop of the recognition accuracy for quieter sounds (e.g., dishwasher). We did not add artificial background noise to affect the SNR since we wanted to be as close to a real-world scenario as possible.

60 The classification results that we obtained at various distances showed that we can achieve good accuracies with one microphone. This was useful, especially for monitoring houses of the elderly, where the number of sensors should be as small as possible.

The two systems are unobtrusive and preserve privacy as the raw audio is 65 immediately deleted after feature extraction and cannot be recovered from the features.

The rest of the paper is organized as follows. Section 2 discusses related work. Section 3 describes the two systems used in our study (signal acquisition, feature extraction, feature selection and classification). The experimental setup 70 and the results are presented in Sections 4 and 5, respectively. Finally, Section 6 concludes the paper.

\section{Related Work}

Audio-based activity recognition has received a lot of attention from researchers in recent years [16, 17. A number of studies have also taken the 75 first steps to characterize the indoor sound environment and the classification of events [18, 19]. While many approaches addressed the problem of audiobased activity recognition in a home environment [20, 21, 22, 23, there is not enough justification for the classifier and feature selection. Most of them used well-known features from the field of speech recognition (e.g., Mel-frequency Cepstral Coefficients (MFCCs)) along with classifiers, such as the k-Nearest Neighbors $(\mathrm{kNN})$, to serve as a proof of concept for indoor audio-based activity classification. Chu et al. 24] showed that increasing the number of audio 
features does not improve the recognition accuracy of a system classifying environmental sounds and suggested new feature selection techniques, such as Matching Pursuit (MP).

Deep Neural Networks (DNNs) are able to extract important information from the raw data without the need for hand-crafted feature extraction and outperform traditional classifiers in many tasks. There is significant research on recognizing single events in monophonic recordings [25] and multiple concurrent events in polyphonic recordings [26]. Different feature extraction techniques [27, hybrid classifiers [28, 29] and very deep neural models [30] have been explored. However, none of these works compared 1D and 2D CNN architectures for ambient sounds.

Another focus of this work is the duration of the signal used with an audiobased event detection system. Previous work [31, 32, 33] has examined the length needed for sufficient recognition accuracy. They used time-frequency features and simple classifiers, such as Support Vector Machines (SVMs) and Hidden Markov Models (HMMs) to test the performance. The proposed approaches work well with datasets that can classify indoor or outdoor environments, however, due to the high variability in the class and the similarity between the different classes, they can fail in a specific AED task (e.g., in kitchen environment).

Finally, there has been extensive research on the effect of the SNR in the presence of background noise [34, 35, 36. Wang et al. 37] performed experiments for various artificially added SNRs (0-10 dB and clean recordings) using different environmental sound datasets and a hierarchical-diving deep belief network. However, all previous work assumed prior knowledge of the SNR, which is not possible in a real-world environment.

\section{Proposed System Architectures}

We propose two approaches for acoustic event detection in an indoor environment. 


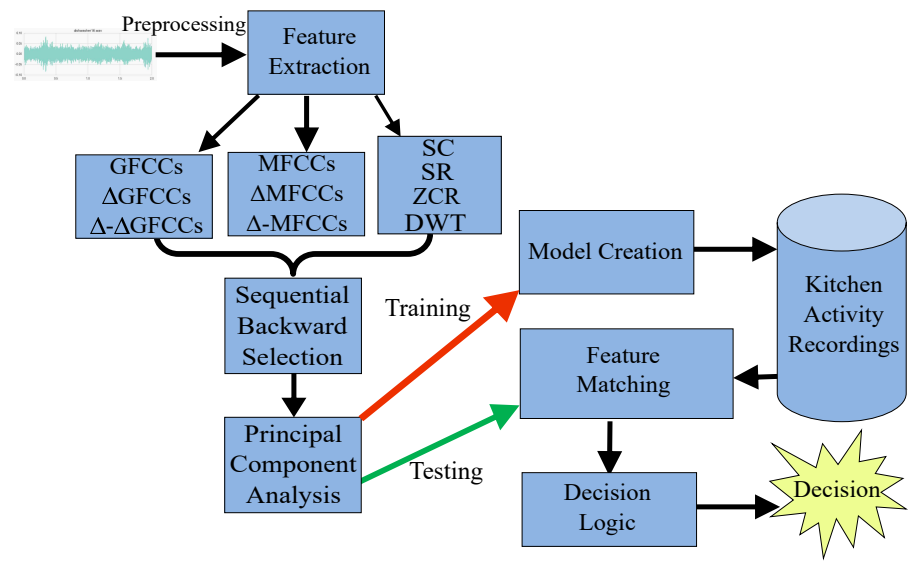

Figure 1: First proposed AED approach.

For the first AED approach (Figure 1), we considered time-domain features (ZCR), frequency-domain features (MFCCs, Gammatone Frequency Cepstral Coeefficients (GFCCs), SR, SC), and time-frequency features (Discrete Wavelet

${ }_{115}$ Transform features). Furthermore, we studied the effect of adding many audio features along with proper feature selection and reduction techniques on recognition accuracy. For classification, we examined well-known classifiers such as k-nearest neighbor, SVM, Random Forest, Extra Trees and Gradient Boosting.

For the second AED approach, we used a CNN trained on mel-spectrogram images (Figure 5). We show that even for a small dataset, a 2-dimensional CNN with 2-dimensional max-pooling (downsamping) layers can provide good recognition accuracy results. The details of the two approaches are given in the following sub-sections.

\subsection{Signal Acquisition}

The success of the signal recording depends on the environment and the placement of the microphone. Ideally the recordings should take place in soundproof studios or labs. However, this is not possible in real life. Therefore, we examined test case scenarios with various types of noises that may occur in a home environment. Three kitchen environments (first author's house, CERTH 

were used for data collection.

In the first step of the preprocessing, we recorded the input signal in stereo at 44,100 Hz (16-bit depth) and then averaged the two channels. This allowed us to use frequencies up to $22,050 \mathrm{~Hz}$, according to the Nyquist criterion. This is sufficient to cover all the harmonics generated by our input signal and removes noise above this range (also not detected by human ear).

\subsection{Data Augmentation}

Environmental audio recordings have various temporal properties. Therefore, we need to make sure that we have captured all the significant information of the signal in both the time and frequency domain. Any environmental signal is a non-stationary signal 24, since it is a stochastic signal and a signal value is not equally probable to occur given another signal value at any time instance.

Previous research [38, 39] showed that data augmentation can significantly improve the performance of a classification system by introducing variability into the original recordings. For this reason, for both AED approaches, we produced two additional recordings from the original ones. Hence the number of audio files was increased from 1,995 to 5,985. First, for each 5 s recording, we added noise with uniform probability distribution. This allowed us to train our system better, since the test audio data in an unknown environment (not used for training) would also include various noises (e.g., different people speaking while performing an activity such as cooking). Second, we re-sampled the original $5 \mathrm{~s}$ recording from $44.1 \mathrm{kHz}$ to $16 \mathrm{kHz}$. Most of the monitored kitchen environment recordings (mixer, dishwasher, faucet, utensils) had a fundamental frequency of around 600-700 Hz. We focused on the harmonics produced by devices such as $\mathrm{kHz}$ was necessary for these classes.

The quality of the data was maintained since i) downsampling removed the frequencies above $16 \mathrm{kHz}$ and did not affect the general recording since the energy of the highest frequencies (above $16 \mathrm{kHz}$ ) was very small and ii) the 


\subsubsection{MFCC: Mel-Frequency Cepstral Coefficients}

MFCCs are one of the most popular features for voice recognition [40]. Figure 2 shows the steps involved in MFCC feature extraction.

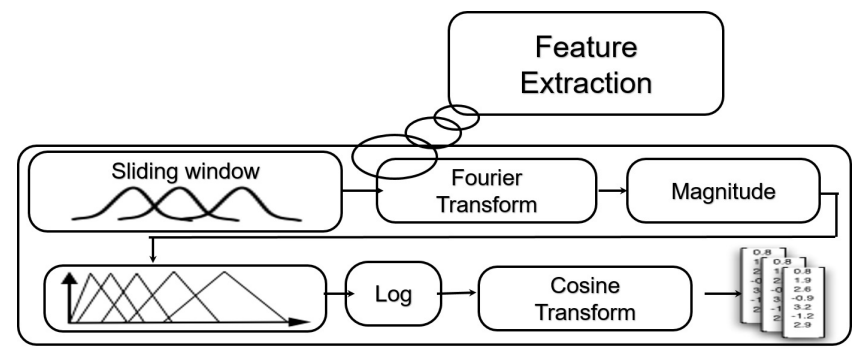

Figure 2: MFCC Feature Extraction

One of the disadvantages of MFCCs is that they are not very robust against

180 additive noise, and so it is common to normalize their values in speech recognition systems to lessen the influence of noise. 
MFCCs are used for voice/speaker recognition. However, the indoor environmental audio signals had significant information at the trajectories of the MFCC coefficients over time. Therefore, we calculated the delta and delta-deltas, also known as differential and acceleration coefficients. The delta coefficients are calculated using Equation 1

$$
\Delta_{c}[m]=\frac{\sum_{i=1}^{K} i(c[m+i]-c[m-i])}{2 \sum_{i=1}^{K} i^{2}}
$$

where $\Delta_{c}[m]$ is the differential coefficient from a frame $m$ computed in terms of the static MFCC coefficients $c[m+i]$ to $c[m-i]$ and $i$ denotes the frame number corresponding to the time-domain frame and $K=2$, meaning that the coefficients are delayed by two frames. The acceleration coefficients are calculated similarly from the deltas.

We used 12 cepstral coefficients, one energy coefficient, 12 delta cepstral coefficients, one delta energy coefficient, 12 double delta cepstral coefficients and one double delta energy coefficient, making a total of 39 MFCC features.

\subsubsection{DWT: Discrete Wavelet Transform}

The DWT provides a compact representation of a signal in time and frequency and can be computed efficiently using a fast, pyramidal algorithm. In the pyramidal algorithm the input signal is analyzed at different frequency bands with different resolution by decomposing it into a coarse approximation and detail information. This is achieved by successive high pass and low pass filtering of the time domain signal. We used an 8-level DWT with the 20-coefficient wavelet family (db20) proposed by Daubechies [41, because of its robustness to noise, and extracted the mean and variance in each sub-band, resulting in 16 (high-frequency) features. The wavelet transform concentrated the signal features in a few large-magnitude wavelet coefficients; hence the coefficients with a small value (noise) could be removed without affecting the input signal quality.

In the kitchen environment signals, high frequency components are present very briefly at the onset of a sound while lower frequencies are present for a long period. 


\subsubsection{ZCR: Zero-Crossing Rate}

In the context of discrete-time signals, a zero crossing is said to occur if successive samples have different algebraic signs. The rate at which zero crossings occur is a simple measure of the frequency content of a signal.

The zero-crossing rate returned a $1 \times 173$ vector for each recording and we calculated the mean and median of each vector, resulting in two ZCR features per recording.

\subsubsection{SR: Spectral Roll-off}

Spectral Roll-off (SR) is defined as the frequency below which a certain percentage ( $85 \%$ - 95\%; depending on the application) of the magnitude distri-

bution of the power spectrum is accumulated. The equation of the feature is given in Equation (2):

$$
\sum_{k=1}^{m} X_{\mathrm{i}}(k)=C \sum_{k=1}^{N} X_{\mathrm{i}}(k)
$$
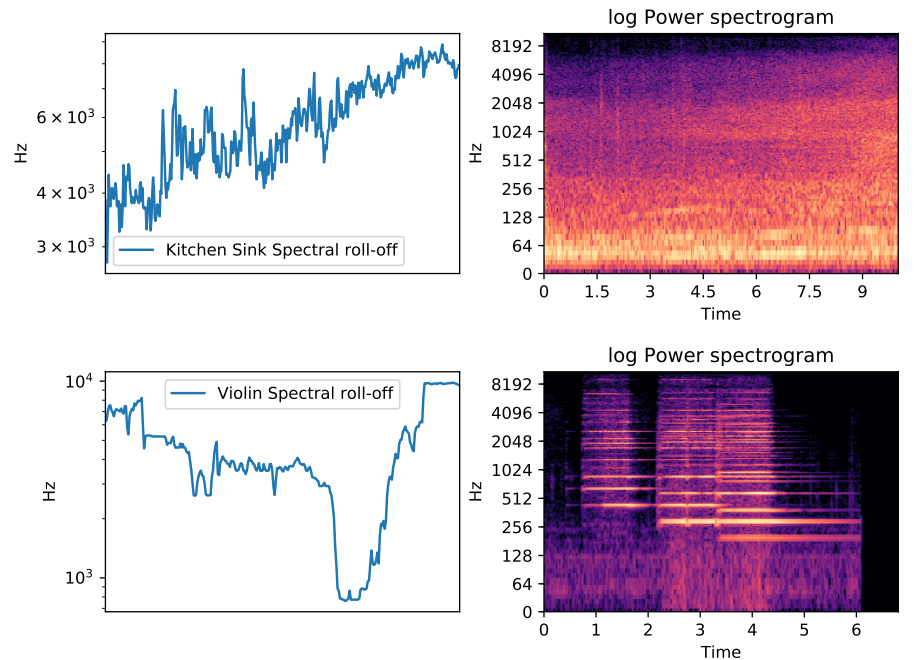

Figure 3: SR comparison between the sound of the kitchen sink (top) and the sound of a violin (bottom)

where the $m$-th DFT coefficient corresponds to the SR of the $i$-th frame, 
$C$ is the percentage of the magnitude distribution of the spectrum and $N$ is the number of frequency bins. We found a threshold of $95 \%$ to be suitable for distinguishing different kitchen sounds. The mean and median of the SR for each recording were calculated and normalized between 0 and 1 .

Figure 3 shows the difference of the SR between a violin recording and the running tap water in the sink. The harmonics of the violin are very distinct in the spectrum, the mean is 0.423 and the median is 0.417 . On the other hand, the mean and median of the kitchen sink sound are 0.811 and 0.803 respectively. These values make sense, since an environmental sound and in particular the running tap water has a widely distributed spectrogram.
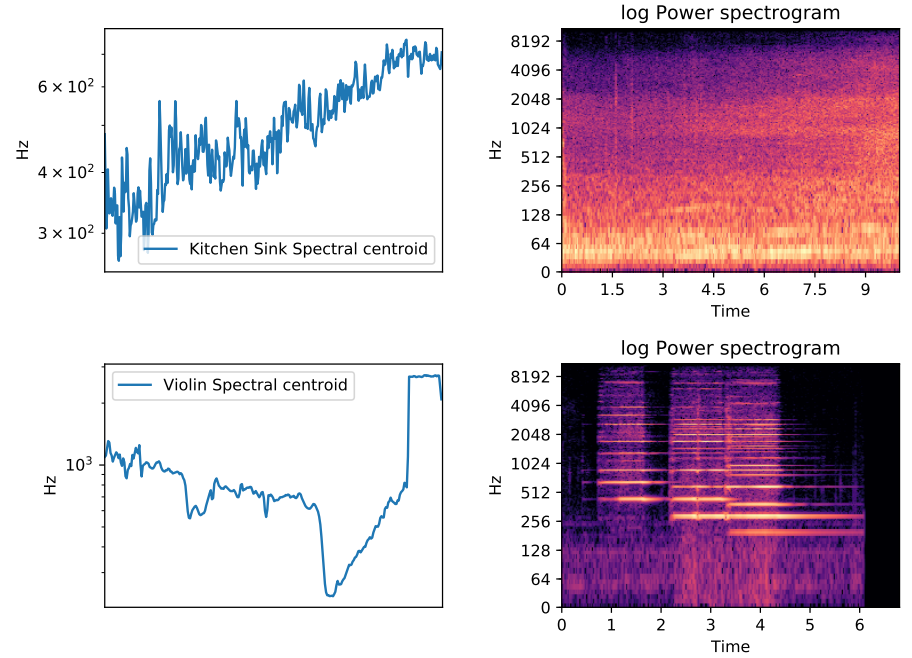

Figure 4: SC comparison between the sound of the kitchen sink (top) and the sound of a violin (bottom)

\subsubsection{SC: Spectral Centroid}

Spectral Centroid (SC) is defined as the "center of gravity" of the spectrum. 235 It is described by Equation (3)

$$
S C=\frac{\sum_{k=1}^{N}(k+1) X_{\mathrm{i}}(k)}{\sum_{k=1}^{N} X_{\mathrm{i}}(k)}
$$


where $X_{i}(k), k=1, \ldots, N$ are the Discrete Fourier Transform (DFT) coefficients of the $i$-th short-term frame and $N$ is the number of frequency bins.

$\mathrm{SC}$ is directly related to the sharpness (high-frequency content) of the sound spectrum. Hence, higher $\mathrm{SC}$ values mean that there is a very bright sound with high frequencies present. The mean and median of the SC for each recording were calculated and normalized between 0 and 1 .

Figure 4 shows a significant difference between the brighter sound of a violin and the more broadband sound of the running water of a kitchen sink. More specifically, for the kitchen sink, where low frequencies are mainly present, the mean is 0.126 and the median 0.113 . On the other hand, the "sharper" sound of the violin, where the harmonics are very distinct at higher frequencies has a mean of 0.383 and a median of 0.366 .

\subsubsection{GFCC: Gammatone Frequency Cepstral Coefficients}

The Gammatone filter-bank consists of a series of band-pass filters, which model the frequency selectivity property of the basilar membrane. The main difference between the MFCC and GFCC is that the Gammatone filter-bank and the cube root are used before taking the DCT versus the triangular filter-bank and the log operation applied in MFCC. Equation (4) describes the calculation of the GFCC:

$$
G F C C_{m}=\sqrt{\frac{2}{N}} \sum_{n=1}^{N} \log \left(E_{n}\right) \cos \left[\frac{\pi n}{N}\left(m-\frac{1}{2}\right)\right], 1 \leq m \leq M
$$

where $E_{n}$ is the energy of the signal in the $n$-th band, $N$ is the number of Gammatone filters and $M$ is the number of GFCC.

We selected 13 GFCC static coefficients, 13 derivatives and 13 doublederivatives coefficients, resulting in 39 features.

\subsection{Feature Selection}

Feature selection was a crucial step for the first AED approach, since we wanted to have a framework that detects activities in real-time. 


\subsubsection{Feature Aggregation}

Out of the 5,985 recordings (original=1,995 and two augmented=3,990), we extracted 100 features per recording $(16(\mathrm{DWT})+2(\mathrm{ZCR})+2(\mathrm{SR})+2$ $(\mathrm{SC})+39$ (GFCC) +39 (MFCC)). Aggregating all the features into a single vector is an important step before passing it to the sequential backward search algorithms and applying the principal component analysis. Feature extraction and classification (using the first AED approach) ran on a Raspberry Pi 3 Model B platform.

\subsubsection{SBS: Sequential Backward Selection}

SBS starts from the whole feature set $X=\left\{x_{i} \mid i=1, \ldots, N\right\}$ and discards the "worst" feature $\left(x^{\prime}\right)$ at each step, such that the reduced set $X-\left\{x^{\prime}\right\}$ gives the maximum value of an objective function $J\left(X-\left\{x^{\prime}\right\}\right)$. Given a feature set SBS gives better results but is computationally more complex than other statistical feature selection methods 42 . With SBS, we reduced the number of features to 17 per recording.

\subsubsection{PCA: Principal Component Analysis}

The central idea of PCA is to reduce the dimensionality of a dataset that consists of many interrelated variables, while retaining as much as possible the variation present in the dataset. We applied PCA to the features given by SBS to reduce the feature space down to two principal components. The principal components were used as input to the classifier.

\subsection{Activity Classification}

For the first AED approach, we compared the performance of a kNN classifier with 5 nearest neighbors, an SVM with a linear and a Radial Basis Function (RBF) kernel, an Extra Trees classifier, a Random Forest and finally the Gradient Boosting classifier.

For the second AED approach, we implemented a CNN based on a modified AlexNet 43 architecture. The CNN was trained on an NVIDIA GeForce GTX $1080 \mathrm{Ti}$ 


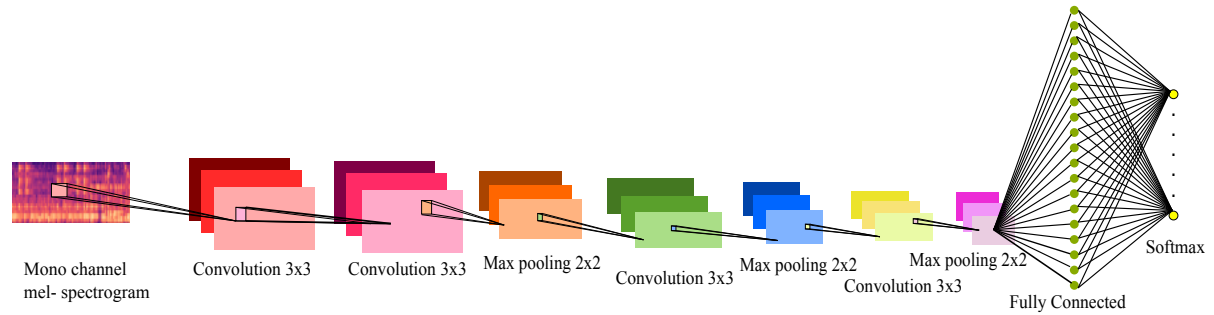

Figure 5: The second proposed AED approach

The CNN consists of 4 convolutional layers (Figure 5). The filter size at each layer increases as a power of two. Specifically the first one has 8 filters, the second 16, the third 32 and the fourth one 64 . The first layer performs convolutions over the spectrogram of the input segment, using $3 x 3$ kernels. The output is fed to a second convolutional layer which is identical to the first. A $2 x 2$ max pooling operation follows the second layer and the subsampled feature maps are fed to two consecutive convolutional layers, each followed by max pooling operations. Each convolution operation is followed by batch normalization [4] of its outputs, before the element-wise application of the exponential linear unit (ELU) activation function [45] to facilitate training and improve convergence time. We selected the ELU activation function based on the results obtained by Clevert et al. 45, where it outperformed other commonly used activation functions (e.g., rectified linear unit (ReLU)), when tested on image datasets using deep neural networks with more than five layers. After each max pooling operation, we apply dropout [46] with an input dropout rate of 0.2 . The number of kernels in all convolutional layers is 5 . The resulting feature maps of the consecutive convolution-max pooling operations are then fed as input to a fullyconnected layer with 128 logistic sigmoid units to which we also apply dropout with a rate of 0.2 , followed by the output layer which computes the softmax function. Classification is obtained through hard assignment of the normalized output of the softmax function

$$
c=\arg \max _{i=1, \ldots, N} y_{i}
$$




$$
y_{i}=\frac{\exp x_{i}}{\sum_{j=1}^{N} \exp x_{j}}
$$

where $N$ is the number of classes and $x_{i}$ is the un-normalized probability for the $i$-th class. We used the Adam [47] optimizer when training our network with an initial learning rate $l_{r}=0.001$ which was reduced by a factor of 0.01 , when there was no validation loss (categorical cross-entropy) improvement for 5 consecutive epochs. This ensured that there was no overfitting in the training. We trained the CNN for 20 epochs.

\section{Experimental Setup}

We recorded sounds of activities using the kitchen setup of Figure 6 (a), where there was no background noise and Figure 6 (b, c) that included background speech sounds and ambient noise of a fan and refrigerator. We also collected sounds for seven classes from Freesound [48].

The first recordings were made in the kitchen of the first author (Figure 6 (a)). Only one person was present at the time of the recordings. For this environment, two smartphones (Samsung Galaxy S5 \& ZTE Nubia Z11 miniS) were placed on the kitchen counter above the dishwasher at an identical position. The main reason for using two smartphones was to capture the same source from two different, off the shelf, microphones. The smartphones were $50 \mathrm{~cm}$ away from the faucet, approximately $50 \mathrm{~cm}$ from the mixer, $1 \mathrm{~m}$ from the oven and approximately $2 \mathrm{~m}$ from the kitchen drawer. For the second set of recordings, the setup was as follows (Figure 6(b)):

1. we used a Raspberry Pi 3 Model B with an MEMS DSP board to record the audio signals

2. the environment was noisier than for the first set of recordings because other researchers were present and speech or other environmental noises were captured more frequently

3. the MEMS board was placed at $1.2 \mathrm{~m}$ from the dishwasher, $1.4 \mathrm{~m}$ from the mixer, $40 \mathrm{~cm}$ from the kitchen faucet and $1.6 \mathrm{~m}$ from the oven. Compared 
(a) Home Kitchen Environment Setup

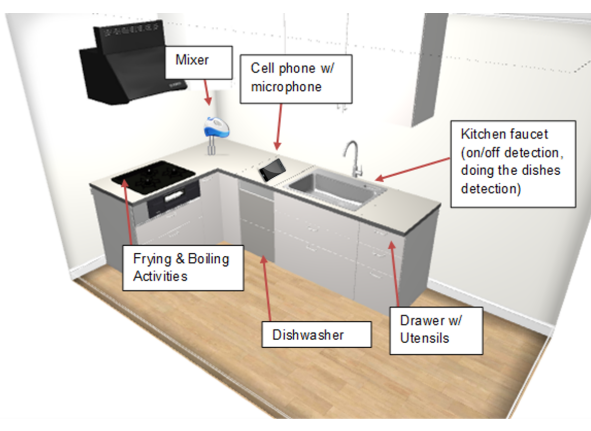

(c) AKTIOS Kitchen Environment Setup

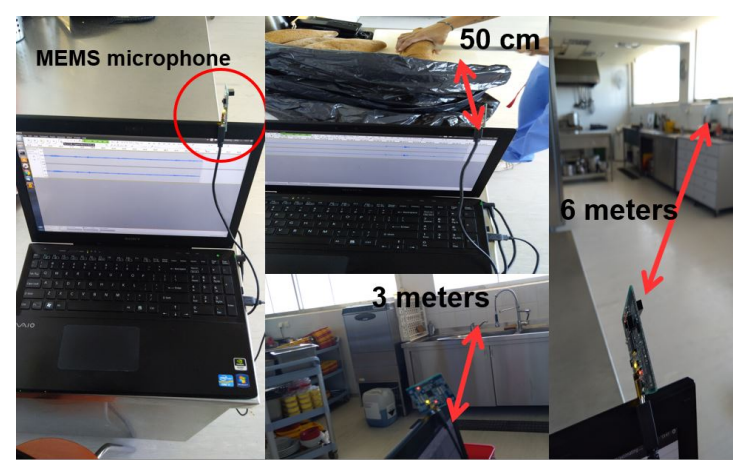

Figure 6: Experimental Setup

to the first recordings, the distances to the appliances were larger to reduce the SNR

Finally, for the third set of recordings (Figure 6(c)), we used a laptop and a MEMS microphone board. For this environment there was a background noise of a fan and a refrigerator. We used the MEMS board to manually adjust the microphone gain $(+6 \mathrm{~dB}$; maximum threshold to avoid clipping when placed within $50 \mathrm{~cm}$ from the cutting board to detect the activity of bread cutting) for the recordings and the laptop to perform real-time classification. The MEMS board was placed in a fixed position on top of the laptop and $3 \mathrm{~m}$ from the kitchen faucet, $3 \mathrm{~m}$ from the dishwasher, $6 \mathrm{~m}$ from the mixer and $50 \mathrm{~cm}$ from 
the cutting board.

Table 1: Number of recordings of each class from different sources

\begin{tabular}{|c|c|c|c|c|}
\hline Classes & $\begin{array}{c}\text { Kitchen } \\
\text { Environment } \\
\text { Figure 6(a) }\end{array}$ & $\begin{array}{c}\text { Kitchen } \\
\text { Environment } \\
\text { Figure 6(b) }\end{array}$ & $\begin{array}{c}\text { Kitchen } \\
\text { Environment } \\
\text { Figure 6(c) }\end{array}$ & Freesound \\
\hline Frying & 160 & 85 & - & 40 \\
\hline Boiling & 160 & 85 & - & 40 \\
\hline Mixer & 160 & 40 & 45 & 40 \\
\hline $\begin{array}{c}\text { Doing the } \\
\text { dishes }\end{array}$ & 160 & 85 & - & 40 \\
\hline Kitchen sink & 160 & 34 & 51 & 40 \\
\hline Dishwasher & 160 & 20 & 65 & 40 \\
\hline Cutting bread & - & - & 285 & - \\
\hline
\end{tabular}

A total of 1,995 audio signals from different activities were collected from the three kitchen environments ( 285 kitchen faucet, 285 boiling, 285 frying, 285 dishwasher, 285 mixer, 285 doing dishes and 285 cutting bread). The setup included the following steps:

- we used data augmentation techniques as described in Section 3.2 to increase the total number of recordings in each class to 855

- Monte Carlo cross-validation was used to randomly split the dataset into training and testing data (80\% training and 20\% testing) and the results (accuracy, precision, recall, F1-score) were averaged over the splits

The total number of recordings from each environment and Freesound is summarized in Table 1

\section{Results}

In this section, we present experiments to assess the performance of our two AED systems. For all of our experiments and comparisons, we applied the same split between the number of training and testing samples, as it is common in

the literature [49. In Section 5.1, we compare several classifiers for the first 
system, we select the one with the highest F1-score and recognition accuracy and compare the performance to that of the second system. In Section 5.2, we study the effect of feature fusion on the recognition rate of the best classifier identified in Section 5.1 (Gradient Boosting). In Section 5.3, we study the recognition accuracy as a function of signal duration. In Section 5.4, we analyze the effect of both the SNR and distance between the microphone and event on the recognition accuracy in an "untrained" environment. In Section 5.5, we examine the response of the second AED system for an activity that was not included in the training set.

5.1. Selection of a traditional classifier for the first AED system and comparison with the second $A E D$ system

Table 2 compares the performance of various classifiers for the selected features. The signals used for this experiment were all the recordings from the three environments mentioned in Section 4 in addition to the Freesound recordings.

Table 2: Classifier Performance Comparison

\begin{tabular}{|l|c|c|c|c|}
\hline \multicolumn{5}{|c|}{ MFCC+GFCC+SR+SC+ZCR+DWT (with augmented data) } \\
\hline \multicolumn{1}{|c|}{ Classifier } & PRECISION & RECALL & F1-SCORE & ACCURACY \\
\hline kNN (5 nearest neighbors) & $78.4 \%$ & $79.4 \%$ & $78.9 \%$ & $79.4 \%$ \\
SVM (linear kernel) & $79 \%$ & $81.2 \%$ & $80.1 \%$ & $83.5 \%$ \\
SVM (RBF kernel) & $84.1 \%$ & $90.1 \%$ & $87 \%$ & $90.9 \%$ \\
Extra Trees & $83.4 \%$ & $85 \%$ & $84.2 \%$ & $89.7 \%$ \\
Random Forest & $88.5 \%$ & $89.1 \%$ & $88.8 \%$ & $91 \%$ \\
Gradient Boosting & $\mathbf{9 0 . 4 \%}$ & $\mathbf{9 0 \%}$ & $\mathbf{9 0 . 2 \%}$ & $\mathbf{9 1 . 7 \%}$ \\
\hline \multicolumn{5}{|c|}{ Mel-Spectrogram (with augmented data) } \\
\hline 2D CNN / $\boldsymbol{w}$ 2D Max-pooling & $\mathbf{9 4 . 6 \%}$ & $\mathbf{9 0 . 9 \%}$ & $\mathbf{9 2 . 7 \%}$ & $\mathbf{9 6 \%}$ \\
\hline 1D CNN /w 1D Max-pooling & $90 \%$ & $89.7 \%$ & $89.8 \%$ & $91.3 \%$ \\
\hline
\end{tabular}

For the Random Forest, we noticed, as the theory suggests, that increasing the number of trees can give a better and more stable performance, hence there is a small chance of overfitting. The number of leaves in the tree had to be small, in order to capture noisy instances in the training dataset. Therefore, we selected 50 samples for each leaf node. For the RBF-based SVM classifier, the highest values for all evaluation measures were found for $\sigma=1$ and $C=0.1$. The parameter $\sigma$ of the RBF kernel handles the non-linear classification and 
the $C$ parameter trades off correct classification of training examples against maximization of the decision functions margin. Finally, for Gradient Boosting we picked 500 estimators. We used the deviance (= logistic regression) loss for classification with probabilistic outputs, since we had a multi-class problem. Another important parameter that affected the classification performance was the learning rate. We performed a grid-search between 0.01 and 0.1 with a 0.01 step and selected 0.05 , which provided the best results. The rest of the parameters from the Scikit-learn [50] library were kept at default. Additionally, as Gradient Boosting is fairly robust to overfitting, the large number of estimators resulted in a better performance, achieving an F1-Score of $90.2 \%$. We obtained good results for boiling, frying, the use of mixer, and also the use of dishwasher. However, the activity of the "running" kitchen faucet was "understood" by our architecture as doing the dishes because some recordings were very similar due to the timing (meaning that no dishes or utensils were "heard" from the microphone).

We compared the performance of the aforementioned classifiers, using standard metrics (accuracy, precision, recall, F1-score). However, in order to comprehend if the best metrics were not affected by the factor of randomness, we performed the Student's T-test for the means of two independent samples of score. We did not assume equal variance between the two samples. According to Table 3, our two best models (Gradient Boosting and 2D-CNN) were statistically different, since they had a p-value of 0.043 , less than the 0.05 threshold. On the other hand the SVM with the RBF kernel, the Extra Trees and the Random Forest were affected by the randomness factor and the classification results could vary, when changing other parameters of the Scikit-learn library. A more reliable characteristic to measure the classification performance, is the Receiver Operating Characteristic (ROC) curve. Figure 7 depicts the ROC curves for all the classifiers that were examined. Classes $0,1,2,3,4,5$ and 6 correspond to boiling, cutting bread, dishwasher, doing the dishes, frying, operating the kitchen faucet and mixer respectively. We noticed that the boiling class was the most easily separable class by all the classifiers. The classes of cutting the bread 
and operating the kitchen faucet were the hardest ones for all the classifiers that we used. This can be explained by the fact that many recordings had the sound of those two particular classes towards the last second of the $5 \mathrm{~s}$ - recording.

In the following experiments, the first AED system will be used with the Gradient Boosting classifier, since it achieved the highest performance characterized by a stable relationship between precision, recall, F1-Score and recognition accuracy.

Table 3: Results of applying the Student's T-test on the classifiers

\begin{tabular}{|c|c|c|}
\hline Classifiers & $\begin{array}{c}\text { Statistically } \\
\text { Different } \\
(\mathrm{p}<\mathbf{0 . 0 5})\end{array}$ & p-value \\
\hline kNN vs SVM Linear & Yes & 0.015462 \\
\hline kNN vs SVM RBF & Yes & $<0.001$ \\
\hline kNN vs Extra Trees & Yes & $<0.001$ \\
\hline kNN vs Random Forest & Yes & $<0.001$ \\
\hline kNN vs Gradient Boosting & Yes & $<0.001$ \\
\hline kNN vs 2D CNN & Yes & $<0.001$ \\
\hline kNN vs $1 \mathrm{D}$ CNN & Yes & $<0.001$ \\
\hline SVM linear vs SVM RBF & Yes & $<0.001$ \\
\hline SVM linear vs Extra Trees & Yes & $<0.001$ \\
\hline SVM linear vs Random Forest & Yes & $<0.001$ \\
\hline SVM linear vs Gradient Boosting & Yes & $<0.001$ \\
\hline SVM linear vs $2 \mathrm{D}$ CNN & Yes & $<0.001$ \\
\hline SVM linear vs $1 \mathrm{D}$ CNN & Yes & $<0.001$ \\
\hline SVM RBF vs Extra Trees & No & 0.558386 \\
\hline SVM RBF vs Random Forest & No & 0.807455 \\
\hline SVM RBF vs Gradient Boosting & No & 0.534736 \\
\hline SVM RBF vs 2D CNN & Yes & $<0.001$ \\
\hline SVM RBF vs 1D CNN & No & 0.360383 \\
\hline Extra Trees vs Random Forest & No & 0.407344 \\
\hline Extra Trees vs Gradient Boosting & No & 0.228362 \\
\hline Extra Trees vs 2D CNN & Yes & 0.001533 \\
\hline Extra Trees vs 1D CNN & No & 0.140385 \\
\hline Random Forest vs Gradient Boosting & No & 0.705960 \\
\hline Random Forest vs 2D CNN & Yes & 0.017268 \\
\hline Random Forest vs 1D CNN & No & 0.495361 \\
\hline Gradient Boosting vs 2D CNN & Yes & 0.043370 \\
\hline Gradient Boosting vs 1D CNN & No & 0.747562 \\
\hline 2D CNN vs 1D CNN & No & 0.110036 \\
\hline
\end{tabular}

In order to highlight the importance of 2D max-pooling, we compared it to 


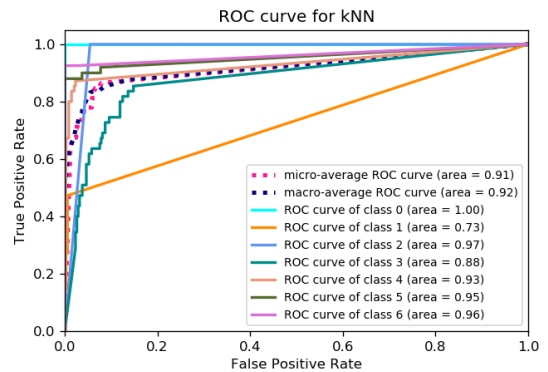

(a) kNN ROC



(c) SVM w/ RBF kernel ROC

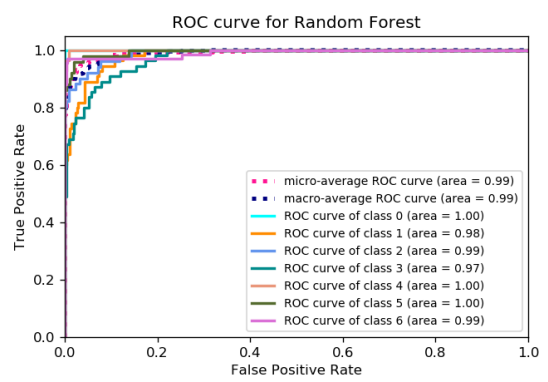

(e) Random Forest ROC



(g) 1D CNN ROC



(b) SVM w/ linear kernel ROC

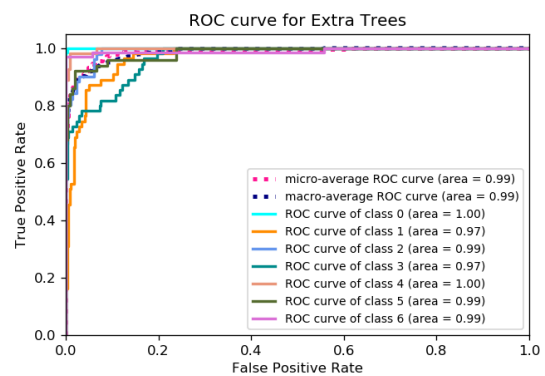

(d) Extra Trees ROC

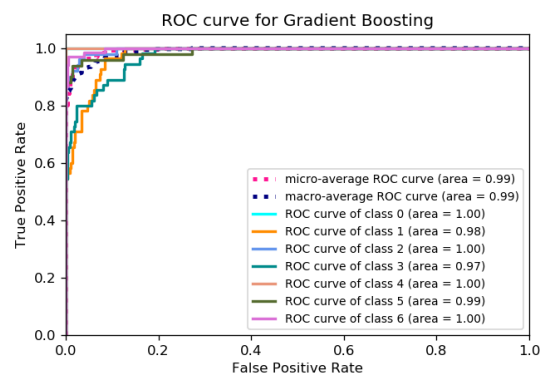

(f) Gradient Boosting ROC

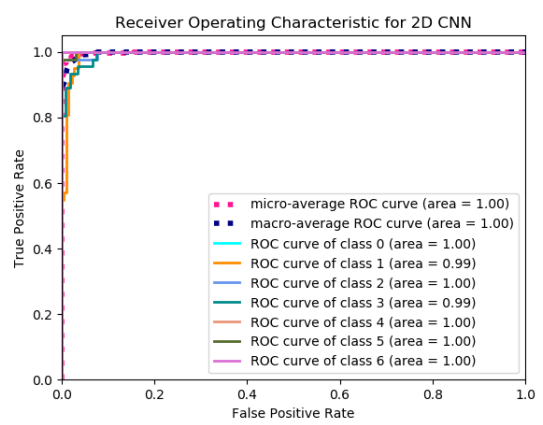

(h) 2D CNN ROC

21

Figure 7: Receiver Operating Characteristic curves for the selected classifiers 
1D max-pooling with a 1D CNN. The input to the 1D CNN network were melspectrograms with 128 bins. The resulting feature matrix input vector to the 1D CNN consisted of 128 mel-band energies in 431 successive frames (number of Fast Fourier Transform (FFT) samples $=1024$ with hop length $=512$, or window size of $20 \mathrm{~ms}$ with a $10 \mathrm{~ms}$ hop size from the next one). The 1D CNN has the same number of filters, kernels, etc. as the 2D CNN (described in Section 3.5). The main differences between the two networks are that kernels change from $3 x 3$ to $3 x 1$, max-pooling from $2 x 2$ to $2 x 1$ and in the Keras code the Conv2D and MaxPooling $2 D$ layers are replaced with the Conv1D and MaxPooling $1 D$ respectively. The $2 \mathrm{D}$ CNN with $2 \mathrm{D}$ max-pooling, was able to capture the spatio-temporal information of the given signal and achieved an F1-Score of 92.7\%. On the other hand, the 1D CNN achieved an F1-Score of $89.8 \%$ only. This showed that the audio signals that were present in the kitchen environment contained important information in the frequency domain.

\subsection{Fusion of features for the first AED approach}

Figure 8 shows how fusing features improves the performance of the first AED approach with the Gradient Boosting classifier. The accuracy rates were calculated for seven feature combinations.

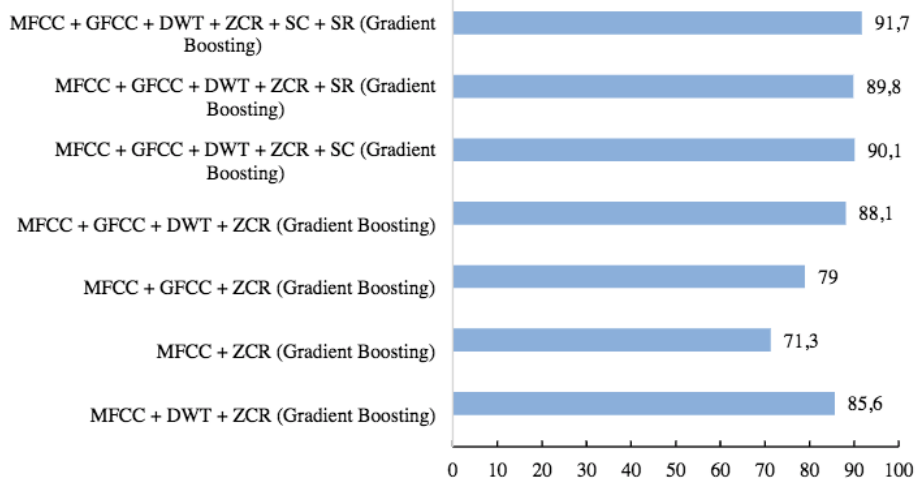

Figure 8: Recognition accuracy for different audio features using Gradient Boosting

Lots of sounds in a kitchen environment have an interchangeable pattern ${ }_{445}$ (bigger/smaller values for odd/even MFCCs). Some mechanical noises (mixer, 
Table 4: Results of applying the Student's T-test on the features

\begin{tabular}{|c|c|c|}
\hline Features & \begin{tabular}{|c|} 
Statistically \\
Different \\
$(\mathrm{p}<0.05)$
\end{tabular} & p-value \\
\hline $\begin{array}{c}\text { MFCCs + GFCCs + DWT + ZCR + SC + SR vs } \\
\text { MFCCs }+ \text { GFCCs }+ \text { DWT }+ \text { ZCR }+ \text { SR }\end{array}$ & No & 0.329507 \\
\hline $\begin{array}{c}\mathrm{MFCCs}+\mathrm{GFCCs}+\mathrm{DWT}+\mathrm{ZCR}+\mathrm{SC}+\mathrm{SR} \mathrm{vs} \\
\mathrm{MFCCs}+\mathrm{GFCCs}+\mathrm{DWT}+\mathrm{ZCR}+\mathrm{SC}\end{array}$ & No & 0.390675 \\
\hline $\begin{array}{c}\mathrm{MFCCs}+\mathrm{GFCCs}+\mathrm{DWT}+\mathrm{ZCR}+\mathrm{SC}+\mathrm{SR} \mathrm{vs} \\
\mathrm{MFCCs}+\mathrm{GFCCs}+\mathrm{DWT}+\mathrm{ZCR}\end{array}$ & No & 0.099163 \\
\hline $\begin{array}{c}\text { MFCCs }+ \text { GFCCs }+ \text { DWT }+\mathrm{ZCR}+\mathrm{SC}+\mathrm{SR} \text { vs } \\
\text { MFCCs }+ \text { GFCCs }+ \text { ZCR }\end{array}$ & Yes & $<0.001$ \\
\hline $\begin{array}{c}\mathrm{MFCCs}+\mathrm{GFCCs}+\mathrm{DWT}+\mathrm{ZCR}+\mathrm{SC}+\mathrm{SR} \mathrm{vs} \\
\mathrm{MFCCs}+\mathrm{ZCR}\end{array}$ & Yes & $<0.001$ \\
\hline $\begin{array}{c}\mathrm{MFCCs}+\mathrm{GFCCs}+\mathrm{DWT}+\mathrm{ZCR}+\mathrm{SC}+\mathrm{SR} \mathrm{vs} \\
\mathrm{MFCCs}+\mathrm{DWT}+\mathrm{ZCR}\end{array}$ & Yes & 0.007203 \\
\hline $\begin{array}{l}\mathrm{MFCCs}+\mathrm{GFCCs}+\mathrm{DWT}+\mathrm{ZCR}+\mathrm{SR} \mathrm{vs} \\
\mathrm{MFCCs}+\mathrm{GFCCs}+\mathrm{DWT}+\mathrm{ZCR}+\mathrm{SC}\end{array}$ & No & 0.906831 \\
\hline $\begin{array}{c}\mathrm{MFCCs}+\mathrm{GFCCs}+\mathrm{DWT}+\mathrm{ZCR}+\mathrm{SR} \mathrm{vs} \\
\mathrm{MFCCs}+\mathrm{GFCCs}+\mathrm{DWT}+\mathrm{ZCR}\end{array}$ & No & 0.498332 \\
\hline $\begin{array}{c}\mathrm{MFCCs}+\mathrm{GFCCs}+\mathrm{DWT}+\mathrm{ZCR}+\mathrm{SR} \mathrm{vs} \\
\text { MFCCs }+\mathrm{GFCCs}+\mathrm{ZCR}\end{array}$ & Yes & $<0.001$ \\
\hline $\begin{array}{c}\mathrm{MFCCs}+\mathrm{GFCCs}+\mathrm{DWT}+\mathrm{ZCR}+\mathrm{SR} \mathrm{vs} \\
\mathrm{MFCCs}+\mathrm{ZCR}\end{array}$ & Yes & $<0.001$ \\
\hline $\begin{array}{c}\mathrm{MFCCs}+\mathrm{GFCCs}+\mathrm{DWT}+\mathrm{ZCR}+\mathrm{SR} \mathrm{vs} \\
\mathrm{MFCCs}+\mathrm{DWT}+\mathrm{ZCR}\end{array}$ & No & 0.084610 \\
\hline $\begin{array}{c}\mathrm{MFCCs}+\mathrm{GFCCs}+\mathrm{DWT}+\mathrm{ZCR}+\mathrm{SC} \mathrm{vs} \\
\mathrm{MFCCs}+\mathrm{GFCCs}+\mathrm{DWT}+\mathrm{ZCR}\end{array}$ & No & 0.427208 \\
\hline $\begin{array}{c}\mathrm{MFCCs}+\mathrm{GFCCs}+\mathrm{DWT}+\mathrm{ZCR}+\mathrm{SC} \mathrm{vs} \\
\mathrm{MFCCs}+\mathrm{GFCCs}+\mathrm{ZCR}\end{array}$ & Yes & $<0.001$ \\
\hline $\begin{array}{c}\mathrm{MFCCs}+\mathrm{GFCCs}+\mathrm{DWT}+\mathrm{ZCR}+\mathrm{SC} \mathrm{vs} \\
\mathrm{MFCCs}+\mathrm{ZCR}\end{array}$ & Yes & $<0.001$ \\
\hline $\begin{array}{c}\mathrm{MFCCs}+\mathrm{GFCCs}+\mathrm{DWT}+\mathrm{ZCR}+\mathrm{SC} \mathrm{vs} \\
\mathrm{MFCCs}+\mathrm{DWT}+\mathrm{ZCR}\end{array}$ & No & 0.065671 \\
\hline $\begin{array}{c}\text { MFCCs + GFCCs + DWT + ZCR vs } \\
\text { MFCCs + GFCCs + ZCR }\end{array}$ & Yes & $<0.001$ \\
\hline $\begin{array}{c}\text { MFCCs }+ \text { GFCCs }+ \text { DWT }+ \text { ZCR vs } \\
\text { MFCCs }+ \text { ZCR }\end{array}$ & Yes & $<0.001$ \\
\hline $\begin{array}{c}\mathrm{MFCCs}+\mathrm{GFCCs}+\mathrm{DWT}+\mathrm{ZCR} \mathrm{vs} \\
\text { MFCCs + DWT + ZCR }\end{array}$ & No & 0.293625 \\
\hline $\begin{array}{l}\text { MFCCs + GFCCs + ZCR vs } \\
\text { MFCCs + ZCR }\end{array}$ & Yes & 0.010891 \\
\hline $\begin{array}{l}\text { MFCCs + GFCCs + ZCR vs } \\
\text { MFCCs + DWT + ZCR }\end{array}$ & Yes & 0.015501 \\
\hline $\begin{array}{c}\text { MFCCs + ZCR vs } \\
\text { MFCCs + DWT + ZCR }\end{array}$ & Yes & $<0.001$ \\
\hline
\end{tabular}


dishwasher) exhibit quite concentrated ZCR distributions, due to some strong fundamental frequencies appearing in the recordings. This served as our motivation to test more time-frequency features in the kitchen recordings. Specifically, when introducing the GFCCs and the DWT, the recognition accuracy was significantly improved. As in the case of the classifiers, the T-test that was performed also on the features (Table 4), using the Gradient Boosting classifier, supported our claim regarding the importance of the DWT and GFCCs in increasing the recognition accuracy, in a noisy environment. MFCCs and ZCR achieved an accuracy of $71.3 \%$. When we added the GFCCs first and DWT second, the accuracy improved to $79 \%$ and $85.6 \%$ respectively. GFCCs use the Equivalent Rectangular Bandwidth (ERB) scale and has finer resolution at low frequencies, which were present at the kitchen environment, compared to the mel-scale that MFCCs use. Additionally, the wavelets were able to separate the fine details of the input signal and increased the recognition accuracy.

\subsection{Recognition accuracy as a function of the audio sample duration}

We studied the impact of segment duration on the accuracy of activity recognition within the kitchen environment. Figure 9 shows that a $3 \mathrm{~s}$ time duration of the input signal is sufficient for accurate activity recognition. For the Gradient Boosting classifier, we noticed an unexpected drop-off for the activity of doing the dishes after the third second. Examination of the confusion matrices revealed that there is a recognition uncertainty of the activity of doing the dishes and the operation of the kitchen sink. After careful listening of all the recordings, we noticed that there were times when the faucet was turned on and only at the last second of the recording an object (plate, utensils) was picked to be washed. On the other hand, the performance of the CNN improved as the audio clips became longer, since it was able to find clear patterns in the mel-spectrogram image. 


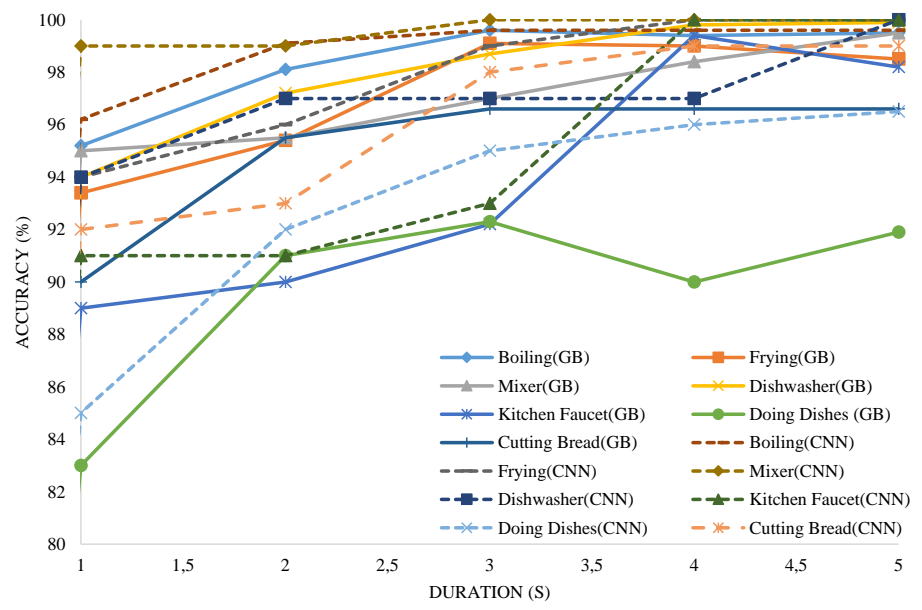

Figure 9: Recognition accuracy (using the Gradient Boosting classifier and the CNN) as a function of the sample duration

\subsection{Dependence of recognition accuracy on certain distance and SNR in a new} environment

We trained both systems in the environments of Figure 6 (a-b) and tested them in the environment of Figure 6 (c). Our training set consisted of 1547 recordings for the seven classes. We tested the systems on the following classes only: dishwasher, mixer, utensils/trays, kitchen faucet. For this experiments, we renamed the class doing the dishes to utensils/trays, since the people in the kitchen rinsed the utensils/trays for a very short period of time and then used the dishwasher. 41 recordings for the activity of moving the utensils/trays were collected in order to test the two AED systems.

Table 5: Confusion matrix using Gradient Boosting for the classes of the framework in a new environment (not included in the training dataset). The distance between the microphone and each activity was $3 \mathrm{~m}$

\begin{tabular}{|c|c|c|c|c|}
\hline & Mixer & Dishwasher & Utensils/Trays & Kitchen Faucet \\
\hline Mixer & 45 & 11 & 0 & 1 \\
\hline Dishwasher & 0 & 48 & 2 & 10 \\
\hline Utensils/Trays & 0 & 1 & 39 & 0 \\
\hline Kitchen Faucet & 0 & 5 & 0 & 40 \\
\hline
\end{tabular}


Table 6: Confusion matrix using CNN for the classes of the framework in a new environment (not included in the training dataset). The distance between the microphone and each activity was $3 \mathrm{~m}$

\begin{tabular}{|c|c|c|c|c|}
\hline & Mixer & Dishwasher & Utensils/Trays & Kitchen Faucet \\
\hline Mixer & 45 & 11 & 0 & 0 \\
\hline Dishwasher & 0 & 46 & 0 & 5 \\
\hline Utensils/Trays & 0 & 2 & 41 & 0 \\
\hline Kitchen Faucet & 0 & 6 & 0 & 46 \\
\hline
\end{tabular}

For this experiment we could not test all seven classes since, i) the recordings from cutting the bread were collected and trained using the setup of that environment, ii) there was no frying activity due to dietary instructions from the elderly care home where the experiment took place and iii) the setup was similar to a restaurant kitchen setup and we could not detect the boiling activity (the microphone was placed at a large distance from the stove). The results (Table 5 and Table 6) show that even with a relatively small training dataset and a distance of $3 \mathrm{~m}$ from the event to be classified, we were able to obtain satisfactory results when testing in a new indoor environment.

Table 7: Recognition accuracy of Gradient Boosting and CNN according to distances and SNRs

\begin{tabular}{|c|c|c|c|c|}
\hline \multirow{2}{*}{ Activities } & $\begin{array}{c}\text { Distance } \\
(\mathbf{m})\end{array}$ & $\begin{array}{c}\text { SNR } \\
(\mathbf{d B})\end{array}$ & $\begin{array}{c}\text { Accuracy } \\
\text { with } \\
\text { Gradient } \\
\text { Boosting } \\
\mathbf{( \% )}\end{array}$ & $\begin{array}{c}\text { Accuracy } \\
\text { with } \\
\text { CNN } \\
\mathbf{( \% )}\end{array}$ \\
\hline Using the & 3 & -27 & 90.2 & 93.4 \\
\cline { 2 - 5 } Kitchen Sink & 0.4 & -10 & 94 & 98.8 \\
\hline Using the & 6 & -11 & 98.5 & 97.1 \\
\cline { 2 - 5 } Mixer & 3 & -8 & 100 & 100 \\
\hline Moving the & 6 & -16 & 91.1 & 95 \\
\cline { 2 - 5 } Utensils/Trays & 3 & -13 & 96.8 & 100 \\
\hline Using the & 3 & -30 & 90.2 & 89.9 \\
\cline { 2 - 5 } Dishwasher & 1 & -25 & 93 & 91.7 \\
\hline
\end{tabular}

The distance between the activity and the microphone affected the recogni- 
tion accuracy. Table 7 shows the SNR and the classification accuracy, using the Gradient Boosting and the CNN, of a set of activities at various distances. The ambient noise of the kitchen at AKTIOS (fan and refrigerator at -32 dB) operating at the time of the experiment dropped the performance of the approaches when increasing the distance from the microphone. The CNN outperformed the Gradient Boosting in most cases. However, in the case of using the mixer when placing the microphone at $6 \mathrm{~m}$ and at $3 \mathrm{~m}$ and $1 \mathrm{~m}$ from the dishwasher, the Gradient Boosting classifier performed better.

\subsection{Tests with an untrained class (coffee machine) using the second AED sys-} tem

For this experiment, we used the MEMS microphone board and a laptop $50 \mathrm{~cm}$ away from a coffee machine to collect 25 recordings of $5 \mathrm{~s}$ each. Out of the 25 recordings, 8 were classified as boiling, since it was the closest match in terms of the audio characteristics of the filter coffee machine. For the remaining 17 the classifier output was discarded because the output probability for each class was below the minimum threshold, set for this experiment to 0.7. More precisely, the class probability was between 0.5 and 0.6 for the boiling class and randomly distributed among the other classes.

\section{Conclusions}

We studied feature extraction, selection, and classification, the effect of signal duration, and SNR at various distances on recognition accuracy in a noisy kitchen environment. Adding more audio features does not necessarily increase the recognition accuracy of the first audio-based event detection system. However, feature selection methods and feature dimensionality reduction techniques, are critical to the success of the system. GFCCs and DWT coefficients significantly increased the recognition accuracy. They outperformed other well-known time-frequency features in the presence of background noise. Furthermore, af- 
provide satisfactory recognition accuracy.The resulting trade-off between time delay and the recognition accuracy was acceptable. The systems were tested in a new environment and provided recognition accuracies above $90 \%$ for appliances that were up to $6 \mathrm{~m}$ away. In most commercial kitchen environments, the distance between the microphone and the target appliance will be smaller. The ability of the CNN to learn from the raw input of mel-spectrogram image increased the classification accuracy compared to the human crafted feature tuned Gradient Boosting. Therefore, one would require minimum pre-processing when deploying a real-world application.

Additionally, even if we had a spatio-temporal classification problem, when adding a basic long-short term memory layer with 64 units and the ReLU activation, after flattening the output of the last convolutional layer, we did not notice an improvement in the classification performance, since it achieved an F1-Score of $92 \%$. This explained that the ambient kitchen sounds do not have very strong temporal information. Since, we did not monitor the activity of a person in a daily scenario, but we captured recordings at various times of the day, the sounds were random and unpredictable.

Finally, in order to check the robustness of our second AED system, we tested it on an untrained class. The softmax layer was able to output probabilities greater than 0.6 for 8 out of the 25 collected recordings, showing that it can be successfully used, $68 \%$ of the time, with a class that is close to a trained one (boiling).

The main limitation of the proposed systems is their inability to distinguish between overlapping events. Since only one acoustic sensor was used, only the loudest event was identified. For instance, when the microphone was placed 6 $\mathrm{m}$ away from the mixer and at the same time $3 \mathrm{~m}$ away from the kitchen faucet, it was able to classify only the activity of the mixer, since the sound of the mixer masked entirely the sound of the running tap water. As future work, we will investigate to which extent multi-channel acoustic recordings are beneficial for the detection of domestic activities in different home environments. To this end, we will use the SINS database [51] which contains more than 200 hours of 
multi-channel recordings from different rooms (living room, kitchen, bathroom, bedroom). Additionally, we plan to keep collecting data in different rooms (e.g., living room, bathroom, etc.), introduce more effects to the signals, such as reverb and echo, and make the collected feature dataset publicly available, in order to help researchers working in this field evaluate their algorithms.

\section{Acknowledgments}

This project has received funding from the European Union's Horizon 2020 research and innovation program under the Marie Skłodowska-Curie grant agreement No. 676157. The authors would also like to thank AKTIOS S.A. Elderly Care Units for allowing real-life testing of the framework.

\section{References}

[1] J. Nehmer, M. Becker, A. Karshmer, R. Lamm, Living assistance systems: An ambient intelligence approach, in: Proceedings of the 28th International Conference on Software Engineering, ICSE '06, ACM, New York, NY, USA, 2006, pp. 43-50.

[2] N.-C. Chi, G. Demiris, A systematic review of telehealth tools and interventions to support family caregivers, Journal of Telemedicine and Telecare 21 (1) (2015) 37-44.

[3] O. D. Lara, M. A. Labrador, A survey on human activity recognition using wearable sensors., IEEE Communications Surveys and Tutorials 15 (3) (2013) 1192-1209.

[4] Y. Lu, Y. Wei, L. Liu, J. Zhong, L. Sun, Y. Liu, Towards unsupervised physical activity recognition using smartphone accelerometers, Multimedia Tools and Applications 76 (8) (2017) 10701-10719.

[5] J. Yin, Q. Yang, J. J. Pan, Sensor-based abnormal human-activity detection, IEEE Transactions on Knowledge and Data Engineering 20 (8) (2008) $1082-1090$. 
[6] L. Meng, C. Miao, C. Leung, Towards online and personalized daily activity recognition, habit modeling, and anomaly detection for the solitary elderly through unobtrusive sensing, Multimedia Tools and Applications 76 (8) (2017) 10779-10799.

[7] A. Bulling, U. Blanke, B. Schiele, A tutorial on human activity recognition using body-worn inertial sensors, ACM Computing Surveys (CSUR) 46 (3) (2014) 33.

[8] Y. Chen, C. Shen, Performance analysis of smartphone-sensor behavior for human activity recognition, IEEE Access 5 (2017) 3095-3110.

[9] A. J. Perez, S. Zeadally, Privacy issues and solutions for consumer wearables, It Professional 20 (4) (2018) 46-56.

[10] P. Kumari, L. Mathew, P. Syal, Increasing trend of wearables and multimodal interface for human activity monitoring: A review, Biosensors and Bioelectronics 90 (2017) 298-307.

[11] D. Giakoumis, G. Stavropoulos, D. Kikidis, M. Vasileiadis, K. Votis, D. Tzovaras, Recognizing daily activities in realistic environments through depth-based user tracking and hidden conditional random fields for mci/ad support, in: European Conference on Computer Vision, Springer, 2014, pp. $822-838$.

[12] I. Kostavelis, D. Giakoumis, S. Malassiotis, D. Tzovaras, Human aware robot navigation in semantically annotated domestic environments, in: International Conference on Universal Access in Human-Computer Interaction, Springer, 2016, pp. 414-423.

[13] J. Chen, A. H. Kam, J. Zhang, N. Liu, L. Shue, Bathroom activity monitoring based on sound, in: International Conference on Pervasive Computing, Springer, 2005, pp. 47-61.

${ }_{605}$ [14] M. Vacher, D. Istrate, F. Portet, T. Joubert, T. Chevalier, S. Smidtas, B. Meillon, B. Lecouteux, M. Sehili, P. Chahuara, et al., The sweet-home 
project: Audio technology in smart homes to improve well-being and reliance, in: Engineering in Medicine and Biology Society, EMBC, 2011 Annual International Conference of the IEEE, IEEE, 2011, pp. 5291-5294.

[15] I. Bisio, A. Delfino, F. Lavagetto, M. Marchese, A. Sciarrone, Genderdriven emotion recognition through speech signals for ambient intelligence applications, IEEE transactions on Emerging topics in computing 1 (2) (2013) 244-257.

[16] K.-Y. Huang, C.-C. Hsia, M.-s. Tsai, Y.-H. Chiu, G.-L. Yan, Activity recognition by detecting acoustic events for eldercare, in: 6th World Congress of Biomechanics (WCB 2010). August 1-6, 2010 Singapore, Springer, 2010, pp. $1522-1525$.

[17] M. Sehili, B. Lecouteux, M. Vacher, F. Portet, D. Istrate, B. Dorizzi, J. Boudy, Sound environment analysis in smart home, Ambient Intelligence (2012) 208-223.

[18] A. Temko, C. Nadeu, D. Macho, R. Malkin, C. Zieger, M. Omologo, Acoustic event detection and classification, Computers in the human interaction loop (2009) 61-73.

[19] H. Lozano, I. Hernáez, A. Picón, J. Camarena, E. Navas, Audio classification techniques in home environments for elderly/dependant people, in: International Conference on Computers for Handicapped Persons, Springer, 2010, pp. 320-323.

[20] J. Chen, A. H. Kam, J. Zhang, N. Liu, L. Shue, Bathroom activity monitoring based on sound, in: Proceedings of the Third International Conference on Pervasive Computing, PERVASIVE’05, Springer-Verlag, Berlin, Heidelberg, 2005, pp. 47-61.

[21] F. Kraft, R. Malkin, T. Schaaf, A. Waibel, Temporal ICA for classification of acoustic events in a kitchen environment, in: INTERSPEECH, Lisbon, Portugal, 2005. 
[22] R. M. Alsina-Pagès, J. Navarro, F. Alías, M. Hervás, homesound: Real-time audio event detection based on high performance computing for behaviour and surveillance remote monitoring, Sensors 17 (4) (2017) 854.

[23] M. Vacher, B. Lecouteux, P. Chahuara, F. Portet, B. Meillon, N. Bonnefond, The sweet-home speech and multimodal corpus for home automation interaction, in: The 9th edition of the Language Resources and Evaluation Conference (LREC), 2014, pp. 4499-4506.

[24] S. Chu, S. Narayanan, C.-C. J. Kuo, Environmental sound recognition with time-frequency audio features, IEEE Transactions on Audio, Speech, and Language Processing 17 (6) (2009) 1142-1158.

[25] D. Barchiesi, D. Giannoulis, D. Stowell, M. D. Plumbley, Acoustic scene classification: Classifying environments from the sounds they produce, IEEE Signal Processing Magazine 32 (3) (2015) 16-34.

[26] G. Parascandolo, T. Heittola, H. Huttunen, T. Virtanen, et al., Convolutional recurrent neural networks for polyphonic sound event detection, IEEE/ACM Transactions on Audio, Speech, and Language Processing 25 (6) (2017) 1291-1303.

[27] H. Eghbal-Zadeh, B. Lehner, M. Dorfer, G. Widmer, CP-JKU submissions for DCASE-2016: A hybrid approach using binaural i-vectors and deep convolutional neural networks, IEEE AASP Challenge on Detection and Classification of Acoustic Scenes and Events (DCASE).

[28] J. Liu, X. Yu, W. Wan, C. Li, Multi-classification of audio signal based on modified svm, in: IET International Communication Conference on Wireless Mobile and Computing (CCWMC 2009), 2009, pp. 331-334.

[29] Y. Xu, Q. Huang, W. Wang, P. Foster, S. Sigtia, P. J. B. Jackson, M. D. Plumbley, Unsupervised feature learning based on deep models for environmental audio tagging, IEEE/ACM Transactions on Audio, Speech, and Language Processing 25 (6) (2017) 1230-1241. 
[30] J. Li, W. Dai, F. Metze, S. Qu, S. Das, A comparison of deep learning methods for environmental sound detection, in: 2017 IEEE International Conference on Acoustics, Speech and Signal Processing (ICASSP), 2017, pp. $126-130$.

[31] J. Portelo, M. Bugalho, I. Trancoso, J. Neto, A. Abad, A. Serralheiro, Nonspeech audio event detection, in: Acoustics, Speech and Signal Processing, 2009. ICASSP 2009. IEEE International Conference on, IEEE, 2009, pp. 1973-1976.

[32] A. J. Eronen, V. T. Peltonen, J. T. Tuomi, A. P. Klapuri, S. Fagerlund, T. Sorsa, G. Lorho, J. Huopaniemi, Audio-based context recognition, IEEE Transactions on Audio, Speech, and Language Processing 14 (1) (2006) 321-329.

[33] J. T. Geiger, B. Schuller, G. Rigoll, Large-scale audio feature extraction and svm for acoustic scene classification, in: Applications of Signal Processing to Audio and Acoustics (WASPAA), 2013 IEEE Workshop on, IEEE, 2013, pp. 1-4.

[34] F. Fuhrmann, A. Maly, C. Leitner, F. Graf, Three experiments on the application of automatic speech recognition in industrial environments, in: International Conference on Statistical Language and Speech Processing, Springer, 2017, pp. 109-118.

[35] H. Erdogan, J. R. Hershey, S. Watanabe, J. Le Roux, Deep recurrent networks for separation and recognition of single-channel speech in nonstationary background audio, in: New Era for Robust Speech Recognition, Springer, 2017, pp. 165-186.

[36] I. McLoughlin, H. Zhang, Z. Xie, Y. Song, W. Xiao, H. Phan, Continuous robust sound event classification using time-frequency features and deep learning, PloS one 12 (9). 
[37] C.-Y. Wang, J.-C. Wang, A. Santoso, C.-C. Chiang, C.-H. Wu, Sound event recognition using auditory-receptive-field binary pattern and hierarchicaldiving deep belief network, IEEE/ACM Transactions on Audio, Speech, and Language Processing 26 (8) (2018) 1336-1351.

[38] J. Salamon, J. P. Bello, Deep convolutional neural networks and data augmentation for environmental sound classification, IEEE Signal Processing Letters 24 (3) (2017) 279-283.

[39] B. McFee, E. J. Humphrey, J. P. Bello, A software framework for musical data augmentation., in: ISMIR, 2015, pp. 248-254.

[40] B. Milner, J. Darch, I. Almajai, S. Vaseghi, Comparing noise compensation methods for robust prediction of acoustic speech features from mfcc vectors in noise, in: 2008 16th European Signal Processing Conference, 2008, pp. $1-5$.

[41] S. Bilgin, O. Polat, O. H. Colak, The impact of daubechies wavelet performances on ventricular tachyarrhythmia patients for determination of dominant frequency bands in HRV, in: 2009 14th National Biomedical Engineering Meeting, 2009, pp. 1-4.

[42] A. Jain, D. Zongker, Feature selection: evaluation, application, and small sample performance, IEEE Transactions on Pattern Analysis and Machine Intelligence 19 (2) (1997) 153-158.

[43] A. Krizhevsky, I. Sutskever, G. E. Hinton, Imagenet classification with deep convolutional neural networks, in: Advances in neural information processing systems, 2012, pp. 1097-1105.

[44] S. Ioffe, C. Szegedy, Batch normalization: Accelerating deep network training by reducing internal covariate shift, in: International Conference on Machine Learning, 2015, pp. 448-456. 
[45] D.-A. Clevert, T. Unterthiner, S. Hochreiter, Fast and accurate deep network learning by exponential linear units (eLUs), arXiv preprint arXiv:1511.07289.

[46] N. Srivastava, G. E. Hinton, A. Krizhevsky, I. Sutskever, R. Salakhutdinov, Dropout: a simple way to prevent neural networks from overfitting., Journal of machine learning research 15 (1) (2014) 1929-1958.

[47] D. P. Kingma, J. Ba, Adam: A method for stochastic optimization, in: Proceedings of the 3rd International Conference for Learning Representations (ICLR-15), 2014.

725 [48] Robinhood76, Kitchen common sounds (2008).

URL https://www .freesound.org/people/Robinhood76/packs/3870

[49] A. Sharif Razavian, H. Azizpour, J. Sullivan, S. Carlsson, Cnn features off-the-shelf: an astounding baseline for recognition, in: Proceedings of the IEEE conference on computer vision and pattern recognition workshops, 2014, pp. 806-813.

[50] 3.2.4.3.5. sklearn.ensemble.gradientboostingclassifier (2019). URL https://scikit-learn.org/stable/modules/generated/ sklearn.ensemble.GradientBoostingClassifier.html\#sklearn . ensemble.GradientBoostingClassifier

[51] G. Dekkers, S. Lauwereins, B. Thoen, M. W. Adhana, H. Brouckxon, T. van Waterschoot, B. Vanrumste, M. Verhelst, P. Karsmakers, The SINS database for detection of daily activities in a home environment using an acoustic sensor network, in: Proceedings of the Detection and Classification of Acoustic Scenes and Events 2017 Workshop (DCASE2017), 2017, pp. 32-36. 